\title{
The Next Generation of Relay-Based PID Autotuners (PART 2): A Simple Relay-Based PID Autotuner with Specified Modulus Margin
}

\author{
Robin DE KEYSER, Oana-Lucia JOITA, Clara-Mihaela IONESCU
}

\begin{abstract}
Ghent University, Eesa-department of Electrical energy, Systems and Automation, Technologiepark 913, B9052 Gent, Belgium (Robain.DeKeyser@UGent.be Oana.Joita@,Yahoo.com ClaraMihaela.Ionescu@,UGent.be)
\end{abstract}

\begin{abstract}
The purpose of this paper is to present the development and evaluation of a novel PID autotuner. Based on prior art results, the algorithm uses the location of the critical point and the value of the critical frequency of the process to impose a user specified robustness on the closed loop. It is shown that the method is easy to apply with few choices left for the user. Nevertheless it is quite successful on systems which are relevant from process engineering point of view.
\end{abstract}

Keywords: autotuner, relay feedback, modulus margin, Nyquist diagram, PID controller, robustness.

\section{INTRODUCTION}

It has long been recognized that industrial control is one of the key technologies to make existing processes economically competitive. In theory, sophisticated control strategies supervisory, adaptive, model predictive control - should be the norm of industrial practice in modern plants. A survey by Desborough and Miller (2001) has shown otherwise: it indicates that $97 \%$ of regulatory controllers are of the proportional-integral-derivative (PID) type and only $32 \%$ of the loops show 'good' performance. One decade has passed since this survey has been published, and the practice of industrial process control is very much the same: PID controllers are widely used and poorly tuned (Yu, 2006).

PID regulators are the backbone of most industrial control systems, thus the problem of determining their best parameters is of great importance in the professional control domain. Process identification is usually very challenging and time consuming. Once the model of a process has been obtained, a controller can be designed by methods that might be application-dependent and, therefore, require a good theoretical background. To simplify this task and to reduce the time required for it, many PID regulators nowadays include autotuning capabilities, i.e. they are equipped with a mechanism capable of computing the 'correct' parameters automatically when the regulator is connected to the plant.

For the automatic tuning of PID controllers, a quasiunlimited number of methods have been proposed. Some of these methods are based on identifying one or more points of the process frequency response, while others are based on the knowledge of some characteristic parameters of the process open-loop step response. Usually these preliminary tests are used to determine a rough model for the process, which is then the basis of an algorithm for tuning the controller parameters. A specific class of autotuners use relay feedback in order to obtain some information on the process frequency response (Yu, 2006; De Keyser and Ionescu, 2010).

Classical approaches such as the Åström-Hägglund (AH) autotuner and Phase Margin (PM) autotuner (Åström et al. 1984, 1992, 2006; Hang et al., 1991), identify the critical point on the process frequency response using such relay feedback. Their advantage is that they are very simple to apply, i.e. few choices are left for the user (which is indeed an advantage if the industrial user is lacking theoretical control engineering insight).

Based on these prior art results, the following section presents some relevant examples for which such simple autotuners will fail to produce good control. A novel method that overcomes the existing drawbacks is then developed, with special care to keep it simple (i.e. few user choices to make). The underlying principles of the proposed algorithm will be provided in the third section of this paper. The evaluation and validation of the novel autotuner on relevant and challenging processes will be presented in detail in the fourth part of the paper. A conclusion section summarizes the main outcome of this work.

\section{LIMITATIONS OF SIMPLE AUTOTUNERS}

For comparison purposes, we use a toolbox for designing PIDs using the full knowledge of the process transfer function. In this paper, the computer aided design (CAD) has been based on the Frequency Response toolbox (FRtool) for Matlab ${ }^{\circledR}$ as described in (De Keyser and Ionescu, 2006). The reader could also use the Root Locus approach (RLtool) in Matlab ${ }^{\circledR}$ or any other model-based PID design method in order to produce a well-tuned PID which will serve as reference for the autotuner evaluation.

Consider the example of the following process: 


$$
P_{1}(s)=\frac{1}{(s+1)^{6}}
$$

The closed loop responses for a setpoint step of value +1 at $\mathrm{t}=0$ and for an input disturbance step of value -1 at $\mathrm{t}=30$ are depicted in Fig. 1 for the AH PID tuner (ref. Appendix) and for the FRtool PID. In this case it can be seen that the autotuner does a good job, performing similarly to the controller designed using the full knowledge of the process.
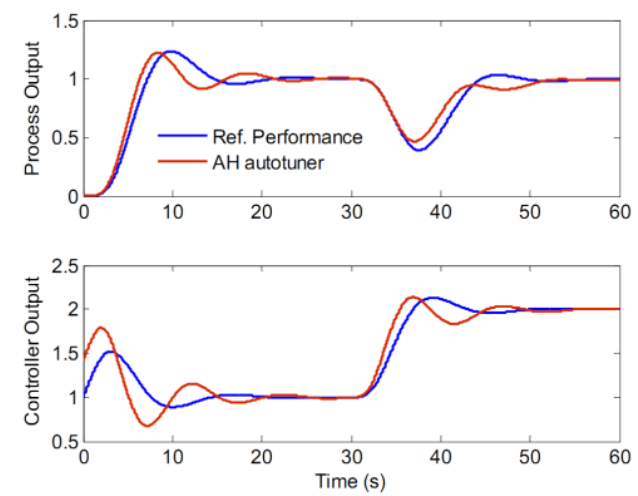

Fig. 1. Closed loop setpoint and disturbance test for the process (1), with the AH tuner and reference PID. AH: $\boldsymbol{K}_{\boldsymbol{P}}=1.4, \boldsymbol{T}_{\boldsymbol{i}}=5.45, \boldsymbol{T}_{\boldsymbol{d}}=1.36$. FRtool: $\boldsymbol{K}_{\boldsymbol{P}}=1, \boldsymbol{T}_{\boldsymbol{i}}=4, \boldsymbol{T}_{\boldsymbol{d}}=1$.

Consider now the case of an integrating process given by:

$$
P_{2}(s)=\frac{32}{s(s+21)(s+3)}
$$
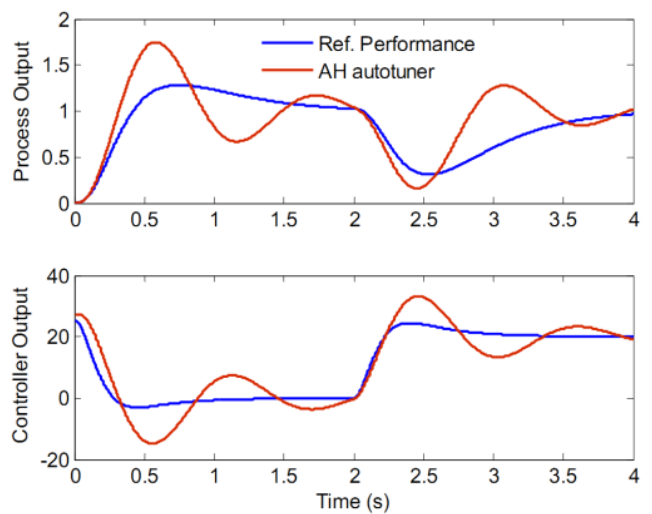

Fig. 2. Closed loop setpoint and disturbance test for the process (2), with the $\mathrm{AH}$ tuner and reference PID. AH: $\boldsymbol{K}_{\boldsymbol{P}}=26, \boldsymbol{T}_{\boldsymbol{i}}=0.4, \boldsymbol{T}_{\boldsymbol{d}}=0.1$. FRtool: $\boldsymbol{K}_{\boldsymbol{P}}=25, \boldsymbol{T}_{\boldsymbol{i}}=0.8, \boldsymbol{T}_{\boldsymbol{d}}=0.2$.

The responses for a setpoint step of value +1 and for an input disturbance step of value -20 are shown in Fig. 2 for the autotuned PID controller and for the reference PID. It can be observed that in this case the autotuner fails to give a satisfactory performance. This behaviour is not specific for the example from (2); it is generally valid for integrating processes; it can also be explained via theoretical insight (Ionescu and De Keyser, 2012).
Let us consider now the case of the Phase Margin (PM) autotuner, which finds the critical point similarly to the $\mathrm{AH}$ relay test and then calculates the controller parameters such that a specified loop phase margin is guaranteed (ref. Appendix).
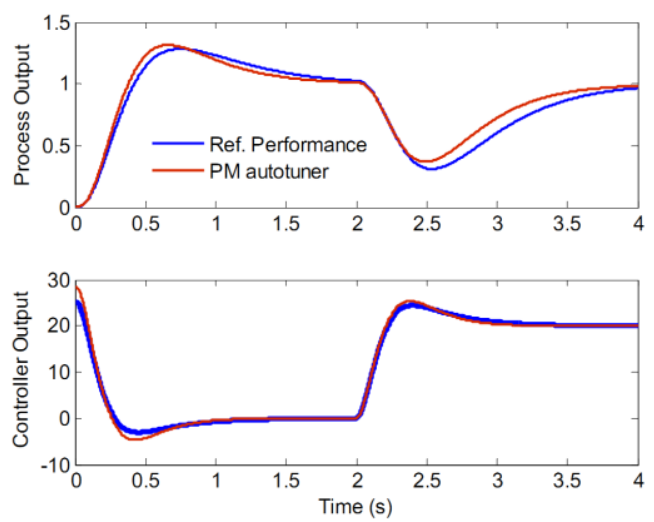

Fig. 3. Closed loop setpoint and disturbance test for the process (2), with the PM tuner and reference PID. PM: $\boldsymbol{K}_{\boldsymbol{P}}=28, \boldsymbol{T}_{\boldsymbol{i}}=0.72, \boldsymbol{T}_{\boldsymbol{d}}=0.18$. FRtool: $\boldsymbol{K}_{\boldsymbol{P}}=25, \boldsymbol{T}_{\boldsymbol{i}}=0.8, \boldsymbol{T}_{\boldsymbol{d}}=0.2$.

First the PM autotuner is tested on the example (2), for which the $\mathrm{AH}$ tuner did not give satisfactory responses. Fig. 3 shows the remarkable performance of the PM autotuner (with a phase margin specification of $50^{\circ}$ ) as compared to the $\mathrm{AH}$ tuner; the results are similar to the model-based PID results.

Now let us consider the following counter-example given by process (3) which has a significant time delay:

$$
P_{3}(s)=e^{-25 s} \frac{2}{(10 s+1)(5 s+1)}
$$

The result for the PM autotuner (designed for a phase margin of $70^{\circ}$, which is a very robust specification) is given in Fig. 4 along with the reference PID designed via FRtool. It can be observed that the PM tuner leads to a PID which makes the control loop unstable.
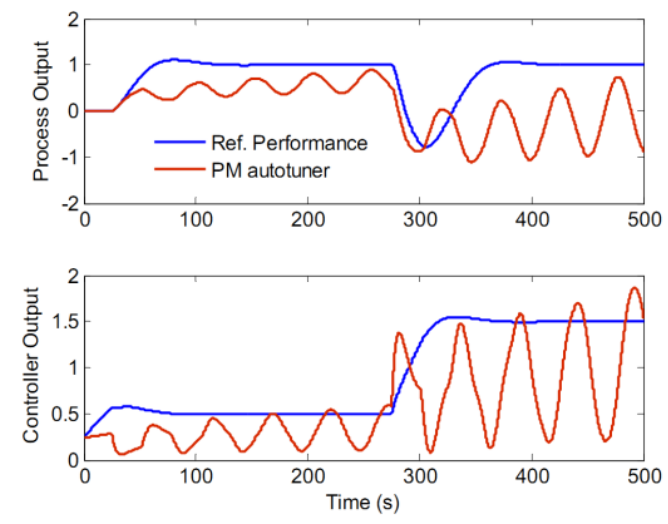

Fig. 4. Closed loop setpoint and disturbance test for the process (3), with the PM tuner and reference PID. PM: $\boldsymbol{K}_{\boldsymbol{P}}=0.24, \boldsymbol{T}_{\boldsymbol{i}}=132, \boldsymbol{T}_{\boldsymbol{d}}=33$. FRtool: $\boldsymbol{K}_{\boldsymbol{P}}=0.25, \boldsymbol{T}_{\boldsymbol{i}}=20, \boldsymbol{T}_{\boldsymbol{d}}=5$. 
Again, this behaviour is not specific for the example (3); it is a generally valid observation that the PM autotuner gives poor results for processes with significant time delay; it can be explained via theoretical insight why this is the case (Ionescu and De Keyser, 2012). An interesting fact to notice is that the $\mathrm{AH}$ autotuner will give excellent results for the system (3).

\section{A NOVEL AUTOTUNING APPROACH}

Controller autotuning is based on the idea of exposing the process to an experiment, during the normal operation. This requires careful design of the excitation signal, to avoid destabilizing the process from its operating point. Traditional relay-based autotuning methods such as AH and PM identify one point on the Nyquist curve of the process $P$ : the intersection of the process beeline with the negative real axis, ref. Fig. 5 (Åström and Hägglund, 2006).

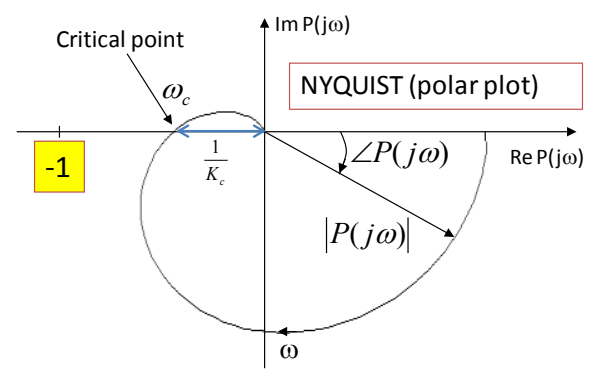

Fig. 5. The process Nyquist plot and the intersection of the beeline with the negative real axis.

Using an appropriate PID controller C, this point is then moved to a specific point in the complex Nyquist plane; e.g. for the AH-tuner, the beeline of $\mathrm{C}^{*} \mathrm{P}$ goes through the specific point $-0.6-0.28 j$ (distance to the point -1 is then 0.5 ) and for the PM-tuner, the beeline of $\mathrm{C}^{*} \mathrm{P}$ goes through the point with modulus ' 1 ' and phase ' $180^{\circ}+$ specified PM'. The insights from (Ionescu and De Keyser, 2012) show that specification of only 1 point in the Nyquist plane might be sufficient for some type of processes, but might as well result in poor (low) modulus margin for other type of processes.

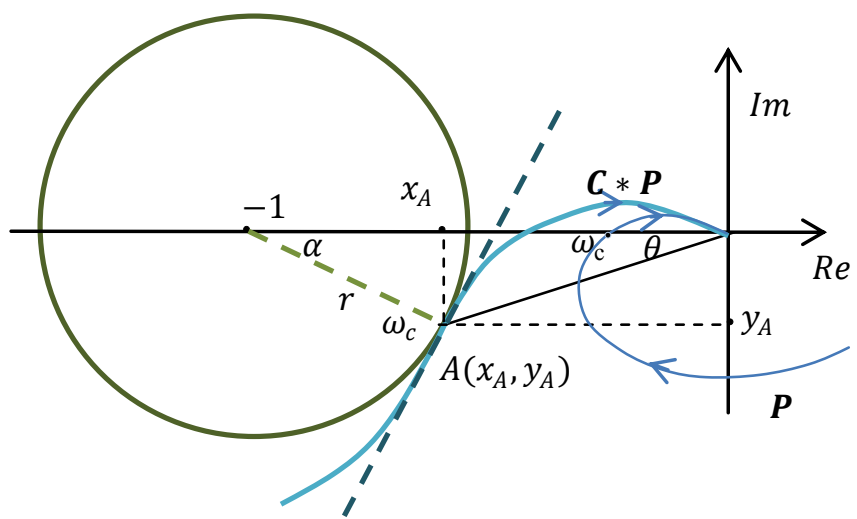

Fig. 6. Novel principle for PID autotuning.
The development of the novel autotuning algorithm is based on imposing a user-specified robustness. The robustness specification can be translated using Nyquist plots as a circle of specified radius $(r)$ around the point -1 as in Fig. 6 ( $r=$ the Modulus Margin, $0<r<1$ ).

In order to obtain a fluent curve for $\mathrm{C}^{*} \mathrm{P}$, going smoothly around the circle with user-specified radius $r$, we impose that at $\omega_{c}$ the $C^{*} \mathrm{P}$ curve will be tangent to the robustness circle, resulting in the following equations at point $\mathrm{A}$ :

$$
\left\{\begin{array}{l}
M_{A} \cdot e^{j \cdot \varphi_{A}}=M_{P C}\left(j \omega_{c}\right) \cdot e^{j \cdot \varphi_{P C}}\left(j \omega_{c}\right) \\
\left.\frac{d M}{d \varphi}\right|_{A}=\left.\frac{d M_{P C}}{d \varphi_{\mathrm{PC}}}\right|_{\omega=\omega_{C}}
\end{array}\right.
$$

That is: the modulus and the phase at point $\mathrm{A}$ should be equal to the modulus and the phase of the process plus controller at $\omega_{c}$. Also the value of the derivative to the circle in A should equal the derivative of the $C^{*} \mathrm{P}$ curve at $\omega_{c}$.

Since we cannot obtain an analytical solution for this system of equations a numerical approach will be presented.

From (4) we get:

$$
\left\{\begin{array}{l}
M_{A}=M_{P C}\left(j \omega_{c}\right)=M_{P}\left(j \omega_{c}\right) \cdot M_{C}\left(j \omega_{c}\right) \\
\varphi_{A}=\varphi_{P C}\left(j \omega_{c}\right)=\varphi_{C}\left(j \omega_{c}\right)+\varphi_{P}\left(j \omega_{c}\right)
\end{array}\right.
$$

The modulus of the process at the critical frequency $M_{P}\left(j \omega_{c}\right)$ is known from the classical relay test (it is $1 / K_{c}$ ). Since $\omega_{c}$ represents the intersection of the process with the negative axis we have that:

$$
\varphi_{P\left(j \omega_{c}\right)}=-\pi
$$

Starting from the typical form of the PID controller

$$
C(j \omega)=K_{P}\left(1+\frac{1}{T_{i} \cdot j \omega}+T_{d} \cdot j \omega\right)
$$

we can write at $\omega_{c}$ in real and imaginary parts:

$$
C\left(j \omega_{c}\right)=K_{P}+\mathrm{j} \cdot K_{P} \frac{T_{d} T_{i} \omega_{c}{ }^{2}-1}{T_{i} \omega_{c}}
$$

obtaining thus the modulus and the phase of the controller:

$$
\begin{aligned}
& M_{C\left(j \omega_{c}\right)}=K_{P} \sqrt{1+\left(\frac{T_{d} T_{i} \omega_{c}^{2}-1}{T_{i} \omega_{c}}\right)^{2}} \\
& \varphi_{C\left(j \omega_{c}\right)}=\operatorname{atan}\left(\frac{T_{d} T_{i} \omega_{c}^{2}-1}{T_{i} \omega_{c}}\right)
\end{aligned}
$$

Replacing (8) and (12) into (7) we have:

$$
\frac{T_{d} T_{i} \omega_{c}{ }^{2}-1}{T_{i} \omega_{c}}=\frac{r \sin (\propto)}{1-r \cos (\propto)}=\tan (\theta)
$$

with $\alpha$ and $\theta$ as defined in Fig.6. 
Considering two identical zeros for the controller $\left(T_{i}=4 T_{d}\right)$ and taking only the positive solution for $T_{d}$ we get:

$$
T_{d}=\frac{M_{A}+r \sin (\propto)}{2 \omega_{c}(1-r \cos (\propto))}
$$

where $M_{A}$ is also a function of $\propto$ derived by applying Generalized Pythagorean Theorem (GPT) for angle $\propto$ in Fig. 6:

$$
M_{A}=\sqrt{r^{2}-2 r \cos (\propto)+1}
$$

Substituting $T_{d}$ in (6) the value for $K_{P}$ is obtained:

$$
K_{P}=\frac{1-r \cos \alpha}{M_{P}\left(j \omega_{c}\right)}
$$

Using again GPT in Fig. 6 we can write $M_{A}$ as a function of $\theta=\varphi-\pi$ :

$$
M_{A}^{2}-2 M_{A} \cos \theta+1=r^{2}
$$

with the solutions:

$$
M_{A 1,2}=\cos \theta \pm \sqrt{r^{2}-\sin \theta^{2}}
$$

Now we can express the variation of the modulus with the phase, which describes the tangent to a circle of radius $r$ around the point -1 :

$$
\frac{d M_{A_{1,2}}}{d \varphi}=\frac{d M_{A_{1,2}}}{d \theta}=-\sin \theta \pm \frac{\sin \theta \cos \theta}{\sqrt{r^{2}-\sin \theta^{2}}}
$$

Applying trigonometric identities in Fig. 6 we have that:

$$
\sin \theta=\frac{r \sin (\alpha)}{M_{A}}, \quad \cos \theta=\frac{1-r \cos (\propto)}{M_{A}}
$$

which allows us to write the solutions of the tangent as a function of $\propto$ :

$$
\frac{d M_{A_{1,2}}}{d \varphi}=\frac{r \sin (\propto)}{M_{A}}\left(-1 \pm \frac{1-r \cos (\propto)}{r|r-\cos (\propto)|}\right)
$$

As it can be seen, the solutions are discontinuous at $r=$ $\cos (\propto)$, where the variation of the modulus with the phase changes sign. Since we only need to work with points on the fourth quadrant of the circle we will need to consider a positive variation for $r<\cos (\propto)$, which is solution 1 , and a negative variation for $r>\cos (\propto)$, which is solution 2 .

In order to find the expression for the tangent to the $\mathrm{C}^{*} \mathrm{P}$ curve we will use the variations of the modulus and phase with frequency.

$$
\left.\frac{d M_{P C}}{d \varphi_{P C}}\right|_{\omega=\omega_{C}}=\frac{\left.\frac{d M_{\mathrm{PC}}}{d \omega}\right|_{\omega=\omega_{C}}}{\left.\frac{d \varphi_{\mathrm{PC}}}{d \omega}\right|_{\omega=\omega_{C}}}=
$$

$$
=\frac{\left.M_{P}\left(j \omega_{C}\right) \frac{d M_{C}}{d \omega}\right|_{\omega=\omega_{C}}+\left.M_{C}\left(j \omega_{c}\right) \frac{d M_{P}}{d \omega}\right|_{\omega=\omega_{C}}}{\left.\frac{d \varphi_{C}}{d \omega}\right|_{\omega=\omega_{C}}+\left.\frac{d \varphi_{P}}{d \omega}\right|_{\omega=\omega_{C}}}
$$

Since we do not have a model for the process we will approximate the derivative of the process frequency response in $\omega_{C}$ using differences:

$$
\begin{aligned}
& \left.\frac{d M_{P}}{d \omega}\right|_{\omega=\omega_{C}}=\frac{\Delta M_{P}}{\Delta \omega}=\frac{M_{P}\left(j \omega_{c}\right)-M_{P}\left(j \omega^{\prime}\right)}{\omega_{c}-\omega^{\prime}} \\
& \left.\frac{d \varphi_{P}}{d \omega}\right|_{\omega=\omega_{C}}=\frac{\Delta \varphi_{P}}{\Delta \omega}=\frac{\varphi_{P}\left(j \omega_{c}\right)-\varphi_{P}\left(j \omega^{\prime}\right)}{\omega_{c}-\omega^{\prime}}
\end{aligned}
$$

where $M_{P}\left(j \omega^{\prime}\right)$ and $\varphi_{P}\left(j \omega^{\prime}\right)$ are the modulus and phase of the process at a frequency $\omega^{\prime}$ which is close to the critical frequency $\omega_{c}$. This can be easily obtained using a relay test with time delay.

By differentiating (11) and (12) with respect to frequency we have:

$$
\begin{gathered}
\left.\frac{d M_{C}}{d \omega}\right|_{\omega=\omega_{C}}=\frac{r \sin (\propto)}{M_{P}\left(j \omega_{c}\right) \omega_{C}} \\
\left.\frac{d \varphi_{C}}{d \omega}\right|_{\omega=\omega_{C}}=\frac{1-r \cos (\propto)}{M_{A} \omega_{C}}
\end{gathered}
$$

Next, by finding iteratively the angle $\alpha^{*}$ for which the error $\left|\frac{d M}{d \varphi}\right|_{A}-\left.\frac{d M_{P C}}{d \varphi_{P C}}\right|_{\omega=\omega_{C}} \mid$ is minimum, we obtain the optimal parameters of the controller for a specified modulus margin $r$ :

$$
\begin{aligned}
K_{P} & =\frac{1-r \cos \left(\alpha^{*}\right)}{M_{P}\left(j \omega_{c}\right)} \\
T_{d} & =\frac{M_{A}\left(\alpha^{*}\right)+r \sin \left(\alpha^{*}\right)}{2 \omega_{c}\left(1-r \cos \left(\alpha^{*}\right)\right)} \\
T_{i} & =4 T_{d}
\end{aligned}
$$

\section{VALIDATION ON ILLUSTRATIVE EXAMPLES}

The algorithm has been validated on many relevant systems and for different robustness specifications. A selection is made in this section. The controllers will be compared against the reference controller for setpoint trajectory following and for input disturbance rejection.

\subsection{Sixth order system}

Let us test the new PID tuner on the $6^{\text {th }}$ order system (1):

$$
P_{1}(s)=\frac{1}{(s+1)^{6}}
$$

The validation on Nyquist curves for two different robustness specifications can be seen in Fig 7. 

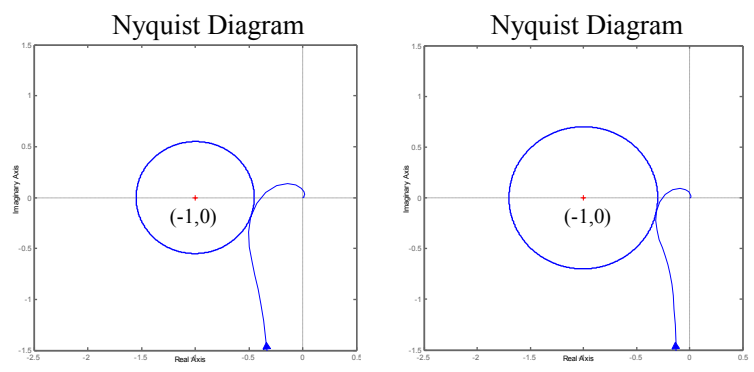

Fig. 7. Process (1); MM=0.55 (left) and 0.70 (right)

The responses for a setpoint step of value +1 and for an input disturbance of value -1 are depicted in Fig. 8 for the novel PID and for a PID controller designed with FRtool using the full knowledge of the process model.

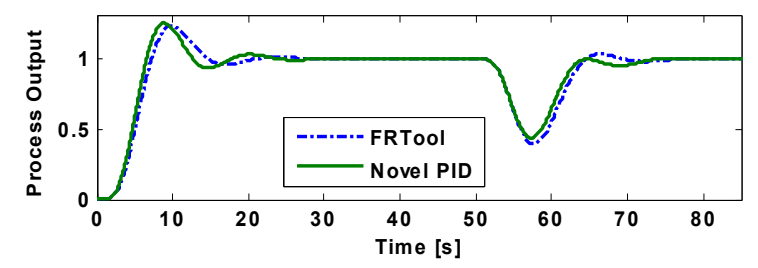

Fig. 8. Process (1); unit step response and disturbance rejection for $\mathrm{MM}=0.55$

It can be seen that there are no notable differences between the 'best design' with FRtool and the novel controller.

\subsection{Third order integrating system}

Reconsider now the case of the integrating process (2):

$$
P_{2}(s)=\frac{32}{s(s+21)(s+3)}
$$

Although difficult to control due to the double integrator in the loop, the new PID tuner has proven to manage different robustness specifications with successful results (Fig. 9).
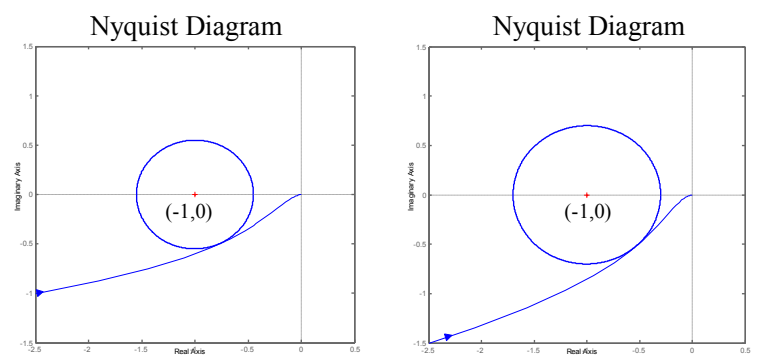

Fig. 9. Process 2; MM=0.55 (left) and 0.70 (right)

The responses for a setpoint step of value +1 and for an input disturbance of value -10 are depicted in Fig. 10.

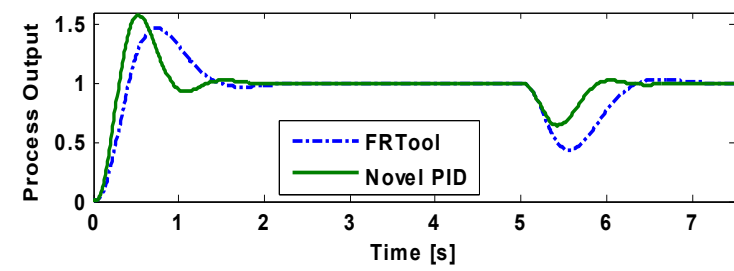

Fig. 10. Process (2); unit step response and disturbance rejection for $\mathrm{MM}=0.55$

Although the FRtool PID has a slightly smaller overshoot than the autotuned controller for the setpoint response, when it comes to disturbance rejection, the novel PID performs remarkably better than the controller designed using the full knowledge of the system. The overshoot can be reduced (at the expense of a less performing disturbance rejection) by increasing the specified MM.

\subsection{Second order system with significant time delay}

Let us reconsider now the time-delay process (3):

$$
P_{3}(s)=e^{-25 s} \frac{2}{(10 s+1)(5 s+1)}
$$

The transfer function has a significant time delay which makes the feedback control for this system a difficult task. The validation on Nyquist curves for robustness specifications of 0.55 and 0.7 can be seen in Fig. 11.
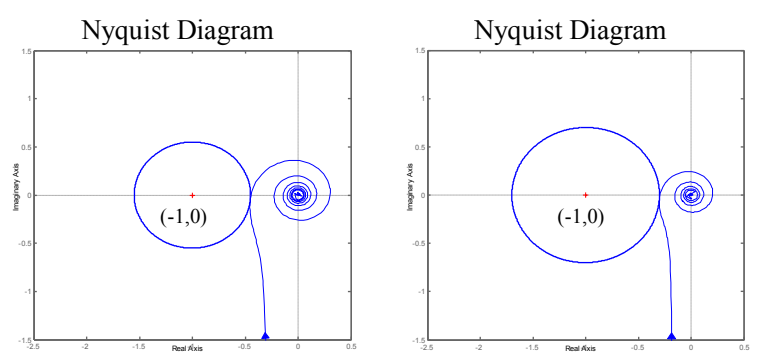

Fig. 11. Process (3); MM=0.55 (left) and 0.70 (right)

The novel autotuner is compared to a PID controller designed with FRtool CAD interface for Matlab. The responses for a setpoint step of value +1 and for an input disturbance step of value -1 are depicted in Fig. 12.

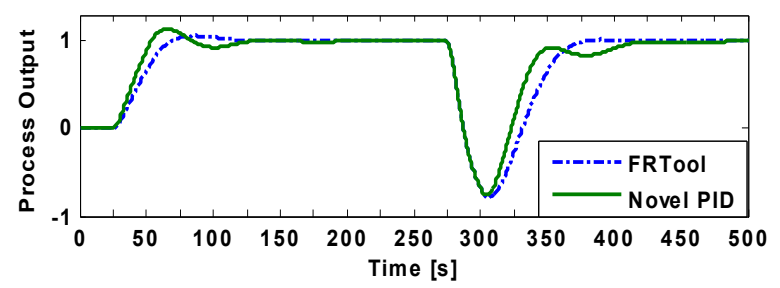

Fig. 12. Process (3); unit step response and disturbance rejection for $\mathrm{MM}=0.55$

Similar conclusions can be taken as for the example in 4.2 . 


\subsection{Integrating process plus time delay}

Since we have noticed that the novel autotuner manages equally well systems with large time delay values and integrating systems, let us consider now the relevant case of a process which presents both particularities:

$$
P_{4}(s)=e^{-0.3 s} \frac{1}{s(s+1)}
$$

Even though the controller is difficult to tune due to the presence of both particularities, the autotuner succeeds in imposing any reasonable robustness specification. Two examples can be seen in Fig. 13.
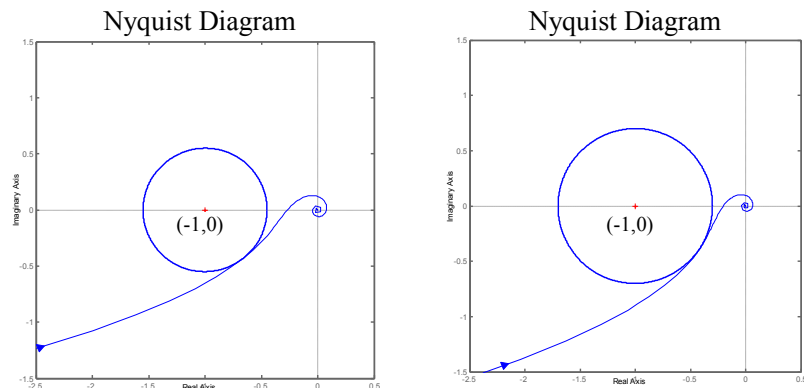

Fig. 13. Process (30); $\mathrm{MM}=0.55$ (left) and 0.70 (right)

The responses for a setpoint step of value +1 and for an input disturbance of value -1 are depicted in Fig. 14 for the novel PID and for a controller designed using the full knowledge of the system. By decreasing the specified value for the MM, the disturbance rejection could be made faster (at the expense of a bigger overshoot).

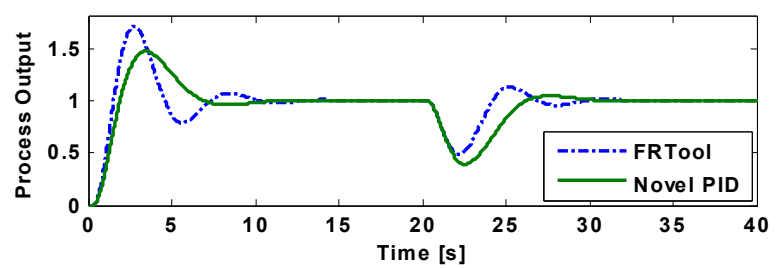

Fig. 14. Process (30); unit step response and disturbance rejection for $\mathrm{MM}=0.55$

\section{CONCLUSIONS}

A novel relay-based PID autotuner has been presented in this paper. The main characteristic of the autotuner is perhaps its simplicity: the user has to specify only 1 design parameter the desired modulus margin - which has a clear interpretation as a trade-off between performance and robustness. The PID autotuner has been successfully tested on several examples that are relevant from process engineering point of view. The simulation results are promising and on-going research is focused on applying the novel PID autotuner on several reallife applications.

\section{REFERENCES}

Åström, K.J., Hägglund, T. (1984), Automatic tuning of simple regulators with specifications on phase and amplitude margins, Automatica 20, 645-651.

Åström, K.J., Hang, C.C., Persson, P., Ho, W.K. (1992), Towards intelligent PID control, Automatica 28, 1-9.

Åström, K.J., Hägglund, T. (2006), Advanced PID Control, ISA.

De Keyser, R., Ionescu, C.M. (2006), FRtool: a frequency response tool for CACSD in Matlab, IEEE Conf. on Computer Aided Control Systems Design, Munich, 22752280.

De Keyser, R., Ionescu, C.M. (2010), A comparative study of three relay-based PID-autotuners, IASTED Asian Conf. on Modeling, Identification and Control (AsiaMIC 2010), Phuket, 303-310.

Desborough, L. D., Miller, R. M. (2001). Increasing customer value of industrial control performance monitoring Honeywell's experience. Chemical Process Control VI Tucson, 172-192.

Hang, C.C., Åström, K.J., Ho, W.K. (1991), Refinements of the Ziegler-Nichols tuning formula, IEE Proc. Design, Control Theory and Appl. 138, 111-118.

Ionescu, C.M., De Keyser R. (2012), The next generation of relay-based PID autotuners (PART1): Some insights on the performance of simple relay-based PID autotuners. IFAC Conf. on Advances in PID Control (PID'12), Brescia Italy.

Yu, C.C. (2006). Autotuning of PID controllers - A relay feedback approach. Springer-Verlag Ltd London.

\section{APPENDIX}

Applying a relay feedback test with relay amplitude $d$, the output of the process will oscillate around the setpoint with a certain output critical amplitude $A_{c}$ and critical period $T_{c}$. The critical gain is then $K_{c}=\frac{4 d}{\pi A_{c}}$. Åström and Hägglund have suggested several ways to calculate a set of PID parameters based on this information. Two of these methods - which are practically interesting because of their simplicity - have been used in section 3 under the designation:

\section{1) $\mathrm{AH}$ method}

The PID parameters are calculated as:

$K_{p}=0.6 K_{c} ; \quad T_{i}=4 T_{d} ; T_{d}=0.125 T_{c}$

In fact, this is the Ziegler-Nichols tuning.

2) PM method

The PID parameters are calculated as:

$$
K_{p}=K_{c} \cos P M ; \quad T_{i}=4 T_{d} ; \quad T_{d}=T_{c} \frac{1+\sin P M}{4 \pi \cos P M}
$$

Here, PM is a user-specified phase margin for the loop. 